\title{
A Scheme of Equitable Distribution of Public Housing in China
}

\author{
Xiao Liu, Huimin Ma, and Jinlong Zhang
}

\begin{abstract}
An increasing number of people have been swarming into the urban in China, resulting in the largest scale of urbanization in the world. In order to alleviate the housing shortage, Chinese government has been building or collecting a large amount of public housing. However, how to distribute the public housing equitably seems a great challenge worthy of consideration. This paper aims to design a scheme to help distribute public housing consistent with the condition in China. We regard identifying the housing preference of the families in need as the crucial part in the distribution process. Meanwhile we also put forward a way to identify the housing preference, and design the principle of two-side matching.
\end{abstract}

Index Terms-Equitable distribution, housing preference, public housing, two-side matching.

\section{INTRODUCTION}

We have witnessed a rapid rate of urbanization, which refers to a move from the rural area into the urban, with the advancement of the economy and technology. In China, nearly 10 million people have immigrated into cities or towns from 2009 to 2012, which arouses a great demand of housing [1]. In consequence, Chinese government has put enormous municipal resources into building or collecting various kinds of public housing. In particular, more than 36 million suits of public housing will be built or collected in the year from 2011 to 2015 [2]. Also, the central government in China plans to raise the occupancy rate of public housing to $20 \%$ at the end of 2015. However, how to distribute so many houses to the families who are really in need seems a great challenge to public housing management agencies, especially in China where the government plays an extremely dominated role in the society.

Many techniques involving ICT or the Internet have been adopted in the business process of public housing management. Currently, the government agencies provide multi-media tools such as portal website for citizens to facilitate their submitting housing applications. They have established many information management systems, most of which are designed to reengineer or solidify the business process, to promote the efficiency of public housing management. On account of the difficulties involved, however, very few systems aim to have the supply side automatically matched with the demand side, namely, the

Manuscript received January 3, 2014; revised April 2, 2014. This work was supported in part by the P.R.C. Minister of Science and Technology under Grant 2012BAJ05B06.

Xiao Liu, Huimin Ma, and Jinlong Zhang are with Huazhong University of Science and Technology, Wuhan, 430074 PRC (e-mail: liu.hsiao.hust@gmail.com). families who are waiting for the distribution of public housing. In contrast, we attempt to design a distribution scheme to solve the dilemma by conducting a research in Huangshi, a city in central China.

\section{EQUitABle Distribution}

Distributing so many suits of public housings not only has technical problems, but also involves different expectations from a variety of stakeholders such as equity and operability. Consequently, we first identify three crucial stakeholders in the process of public housing distribution: applicant family, which refers to the family who needs help from the government to promote their living condition; public housing, which represents the apartment built by the government and to be distributed to the applicant families; housing agency, which is usually an administrative department charging of public housing's management. The challenge for housing agency lies in distributing the public housing to the applicant families equitably.

But what is equity? How to distribute public housing equitably? The definition of equity varies in different disciplinary sectors, and even is not consistent with each other in the same disciplinary sector. Thus, different schemes are put forward according to various definitions. This paper regards equity as a public housing distribution process in which the following two conditions are met: personality and priority.

Personality. We should not regard applicant families as the same ones in that each of them has its own housing preference. Therefore, the scheme may not satisfy the housing demand sufficiently if families' preferences are ignored. For example, assuming that family $A$ prefers housing $C$ to housing $D$, and family $B$ prefers housing $D$ to housing $C$; but the scheme distributes housing $C$ to family $B$, and assigns housing $D$ to family $A$. All of these families do not obtain their preferable housing. Previous studies discussed this point extensively, especially in the case of Stable Marriage Problem [3]. So we believe that an equitable distribution should take as many housing preferences of applicant family into account as possible.

Priority. The heterogeneity in applicant families does not merely mean that they may have different housing preferences. Furthermore, they also differ from others in priorities. As a limited resource, public housing should cover the group in an emergency. Though having different regulations about the distribution, all cities realize that it is necessary to identify the families who anxiously expect to get social welfare from the perspective of equity. Unfortunately, many researchers on distribution or matching overlook the 
fact more or less that sometimes the priority should be strictly complied with.

\section{HOUSING PREFERENCE}

The information housing agency collects is mainly about the basic family condition and merely involves the housing preference. Accordingly, we need to collect some other data to describe housing preference as a supplement.

Housing preference reflects the expectation of the applicant family for public housing. Namely, it describes applicant family's ideal point for public housing. Theoretically, the applicant family has an expectation for each housing attribute, such as location, floor height, orientation, and dwelling unit. However, at least more than 100 statistical attributes are given by Chinese authority according to 'Design code for residential buildings' issued. Thus we should select some crucial attributes based on the order of which we construct the applicant family's housing preference.

After the interviews with the applicant families and officers in the housing agency, we adopt five crucial attributes of public housing in this scheme: location, living space, rental price, floor height, and surroundings. Namely, the expectation and preference order of an applicant family for these five crucial attributes of public housing jointly define its housing preference.

We ask applicant families to fill in their expectations and order preferences for the crucial attributes when collecting data. It is convenient for applicant family to choose a proper item rather than to answer open questions. In this way, furthermore, we can easily gather data online from a very large sample size. Thus, we delivered questionnaires to collect housing preferences in Huangshi. These attributes and their optional items are shown in Table I.

In China the applicant family especially cares about the location, whose optional items include district, sub-district, and community information. Applicant family should choose their preferable district, sub-district and community in the city. Living space and rental price are also common factors when considering a resident building. In Huangshi, public housing is classified into 6 types respectively according to living space or rental price: $15-30 \mathrm{~m}^{2}, 30-45 \mathrm{~m}^{2}, 45-60 \mathrm{~m}^{2}$, 60-75 $\mathrm{m}^{2}$ and no less than $75 \mathrm{~m}^{2}$; or no less than CNY100, no less than CNY200, no less than CNY300, no less than CNY400, and no less than CNY500. Many applicant families also take floor height of public housing into account, especially those who have disabled or senile members. The optional items of floor height include first floor, low floor, medium floor, high floor and top floor. With regard to surroundings, it refers to public facilities in this context and consists of four kinds of facilities: educational facilities, medical facilities, transport facilities and living facilities. The applicant family can choose one or more kinds of facilities as expected surroundings. In addition, we also need to record the preference order of applicant family to these five crucial attributes. For instance, an applicant family's housing preference in Huangshi can be described as follows: a high-floor housing surrounded with educational facilities and medical facilities located in Nanyue Community, Hongqiqiao Sub-district, Huangshigang District, with living space about 60-75 $\mathrm{m}^{2}$ and rental price no less than CNY 300. Meanwhile, the preference order of these crucial attribute is first location, then rental price, then living space, then floor height, and the last is surroundings. Particularly, we set default values according to the local rules for those whose housing preference cannot be expressed directly for some special reasons. To simplify the model, we exclude the rare case in which the applicant family has no special request to a certain attribute.

TABLE I: THE CRUCIAL ATTRIBUTES OF PUBLIC HOUSING

\begin{tabular}{|c|c|}
\hline Crucial Attribute & Optional items \\
\hline Location & $\begin{array}{l}\text { Which Community } \\
\text { Which Sub-district } \\
\text { Which District }\end{array}$ \\
\hline Living space & $\begin{array}{c}15-30 \mathrm{~m}^{2} \\
30-45 \mathrm{~m}^{2} \\
45-60 \mathrm{~m}^{2} \\
60-75 \mathrm{~m}^{2} \\
\text { No less than } 75 \mathrm{~m}^{2}\end{array}$ \\
\hline Rental price & $\begin{array}{l}\text { No less than CNY100 } \\
\text { No less than CNY200 } \\
\text { No less than CNY300 } \\
\text { No less than CNY400 } \\
\text { No less than CNY500 }\end{array}$ \\
\hline Floor height & $\begin{array}{l}\text { First floor } \\
\text { Low floor } \\
\text { Medium floor } \\
\text { High floor } \\
\text { Top floor }\end{array}$ \\
\hline Surroundings & $\begin{array}{c}\text { educational facilities } \\
\text { medical facilities } \\
\text { transport facilities } \\
\text { living facilities }\end{array}$ \\
\hline
\end{tabular}

\section{Two-Side MATCHING}

We label the applicant families as $F$, and the number of the families as $m . F_{i}$ represents a specific applicant family among the set $F$. $H$ represents public housing, whose number is $n$, and $H_{j}$ is one of public housing among the set $H$.

$$
\begin{gathered}
F=\left\{F_{1}, F_{2}, \cdots, F_{i}, \cdots, F_{m}\right\}, i \in[1, m] \\
H=\left\{H_{1}, H_{2}, \cdots, H_{j}, \cdots, H_{n}\right\}, j \in[1, n]
\end{gathered}
$$

This study conducts two-side matching based on the housing preference of applicant family and the real value of public housing. We define $\alpha_{i}, \beta_{i}, \gamma_{i}, \delta_{i}, \varepsilon_{i}$ as the expectation of applicant family $F_{i}$ for location, living space, rental price, floor height and surroundings. And $a_{j}, b_{j}, c_{j}, d_{j}, e_{j}$ represents the real value of public housing $H_{j}$ for each attribute respectively.

The ordinal value of $F_{i}$ to location in preference order is labeled as $v_{i 1}$. Similarly, the ordinal value of $F_{i}$ to living space, rental price, floor height and surroundings are $v_{i 2}, v_{i 3}$, $v_{i 4}, v_{i 5}$ respectively. 


$$
\begin{gathered}
v_{i 1}, v_{i 2}, v_{i 3}, v_{i 4}, v_{i 5} \in\{1,2,3,4,5\} \\
v_{i x} \neq v_{i y}, x \neq y \\
x, y \in\{1,2,3,4,5\}
\end{gathered}
$$

\section{A. Matching Degree}

Matching degree measures the effect of matchmaking between applicant family and public housing. We define $d_{i j}$ as matching degree between family $F_{i}$ and housing $H_{j}$. Since housing preference is characterized by five crucial attributes, the matching degree depends on five sub-matching degree of these attributes.

We define the sub-matching degree function of location as $P_{1}\left(\alpha_{i}, a_{j}\right)$, and $P_{2}\left(\beta_{i}, b_{j}\right), P_{3}\left(\gamma_{i}, c_{j}\right), P_{4}\left(\delta_{i}, d_{j}\right), P_{5}\left(\varepsilon_{i}, e_{j}\right)$ are the functions of living space, rental price, floor height, and surroundings respectively. Each crucial attribute has a weight to reflect its ordinal position in the sequence, and we label the weight functions of location, living space, rental price, floor height and surroundings as $w\left(v_{i 1}\right), w\left(v_{i 2}\right), w\left(v_{i 3}\right), w\left(v_{i 4}\right)$, $w\left(v_{i 5}\right)$. As a result, matching degree $d_{i j}$ can be presented in (1).

$$
\begin{aligned}
& d_{i j}=w\left(v_{i 1}\right) \times P_{1}\left(\alpha_{i}, a_{j}\right)+w\left(v_{i 2}\right) \times P_{2}\left(\beta_{i}, b_{j}\right) \\
& +w\left(v_{i 3}\right) \times P_{3}\left(\gamma_{i}, c_{j}\right)+w\left(v_{i 4}\right) \times P_{4}\left(\delta_{i}, d_{j}\right) \\
& +w\left(v_{i 5}\right) \times P_{5}\left(\varepsilon_{i}, e_{j}\right)
\end{aligned}
$$

All sub-matching degree functions have two parameters: the expectation of applicant family and the real value of public housing for a certain crucial attribute. Now we will present values of these sub-matching degree functions in all kinds of conditions according to the local rules in Huangshi.

Sub-matching degree of location depends on the consistency of expectation and real value on this attribute. The degree equals to full scores if the expected addresses of community, sub-district and district are the same with the real address respectively, and declines with the difference between expectation and real value. We define the value of district in $\alpha_{i}$ as $\alpha_{i 1}$, the sub-district as $\alpha_{i 2}$, and the community as $\alpha_{i 3}$. Similarly, the value of district in $a_{j}$ is $a_{j 1}$, the sub-district is $a_{j 2}$, and the community is $a_{j 3}$. Then $P_{1}\left(\alpha_{i}, a_{j}\right)$ is presented in (2).

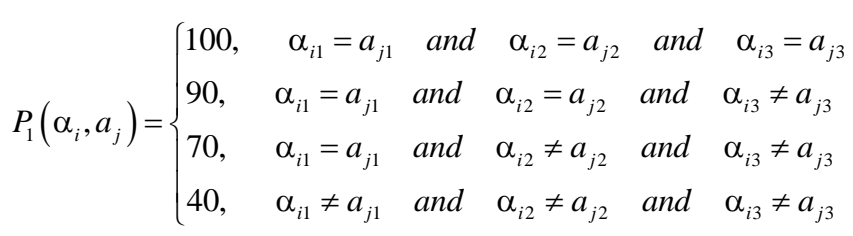

Sub-matching degree of living space also depends on the difference between the expectation and real value. Less difference means a higher degree value. Let $\beta_{i}$ represent an interval value, with the left boundary value $\beta_{i 1}$ and the right boundary value $\beta_{i 2}$. Theoretically, the utility increases with the growth of living space. However, excessive supply of public housing means wasting and unevenness given that it is a kind of scarce and limited resource. Hence, the utility of appropriate supply is higher than that of excessive supply. Then $P_{2}\left(\beta_{i}, b_{j}\right)$ is presented in (3).

$$
P_{2}\left(\beta_{i}, b_{j}\right)= \begin{cases}100, & b_{j} \in\left[\beta_{i 1}, \beta_{i 2}\right] \\ 90, & b_{j}>\beta_{i 2} \\ 85, & 0<\beta_{i 1}-b_{j}<5 \\ 80, & 5 \leq \beta_{i 1}-b_{j}<10 \\ 75, & 10 \leq \beta_{i 1}-b_{j}<15 \\ 70, & 15 \leq \beta_{i 1}-b_{j}<20 \\ 60, & 20 \leq \beta_{i 1}-b_{j}<25 \\ 40, & \beta_{i 1}-b_{j} \geq 25\end{cases}
$$

Similar with that of living space, expectation of rental price $\gamma_{i} \in\{100,200,300,400,500\}$ is a set of discrete values rather than an interval value. So $P_{3}\left(\gamma_{i}, c_{j}\right)$ is presented in (4).

$$
P_{3}\left(\gamma_{i}, c_{j}\right)= \begin{cases}100, & c_{j} \leq \gamma_{i} \\ 95, & 0<c_{j}-\gamma_{i}<50 \\ 90, & 50 \leq c_{j}-\gamma_{i}<100 \\ 80, & 100 \leq c_{j}-\gamma_{i}<150 \\ 70, & 150 \leq c_{j}-\gamma_{i}<200 \\ 60, & 200 \leq c_{j}-\gamma_{i}<250 \\ 40, & c_{j}-\gamma_{i} \geq 250\end{cases}
$$

Though varying with the difference between expectation and real value on this attribute, sub-matching degree of floor height does not show a linear relationship with that difference. Floor height has five types: first floor, low floor, medium floor, high floor and top floor. Both the expectation value and the real value of height floor vary from first floor to top floor. We label first floor as $f_{1}$, and low floor, medium floor, high floor and top floor as $f_{2}, f_{3}, f_{4}, f_{5}$ respectively. And $P_{4}\left(\delta_{i}, d_{j}\right)$ is presented in (5).

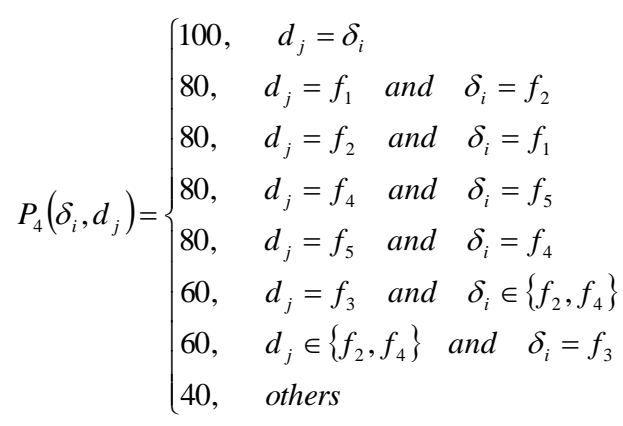

$$
\begin{aligned}
& \delta_{i}, d_{j} \in\left\{f_{1}, f_{2}, f_{3}, f_{4}, f_{5}\right\}
\end{aligned}
$$

Sub-matching degree of surroundings depends on the items chosen and the real value. We label educational facilities, medical facilities, transport facilities and living facilities as $\varepsilon_{i 1}, \varepsilon_{i 2}, \varepsilon_{i 3}$ and $\varepsilon_{i 4}$ respectively, and the real values of housing $H_{j}$ on these four items are $e_{j 1}, e_{j 2}, e_{j 3}$, $e_{j 4}$. All these values range from 40 to 100 . Let $p$ denotes 
the number of the items being chosen. And $P_{5}\left(\varepsilon_{i}, e_{j}\right)$ is presented in (6).

$$
\begin{aligned}
\varepsilon_{i} & \subseteq\left\{\varepsilon_{i 1}, \varepsilon_{i 2}, \varepsilon_{i 3}, \varepsilon_{i 4}\right\} \\
e_{j} & \subseteq\left\{e_{j 1}, e_{j 2}, e_{j 3}, e_{j 4}\right\} \\
P_{5}\left(\varepsilon_{i}, e_{j}\right) & =\frac{\sum e}{p}, e \in e_{j}, p \leq 4, p \in N^{+}
\end{aligned}
$$

Each sub-matching degree has a different weight to reflect its significance in the preference order. In this scheme, we first convert preference order into ordinal value, then transform ordinal value to importance value, which will be replaced by weight through a serial of mathematical manipulations.

For family $F_{i}$, we define the weight function as $w\left(v_{i}\right)$, and the importance function as $s\left(v_{i}\right)$.

$$
v_{i} \in\left\{v_{i 1}, v_{i 2}, v_{i 3}, v_{i 4}, v_{i 5}\right\}
$$

The importance function has following characteristics: a). $\left.s\left(v_{i}\right)>0 ; \mathrm{b}\right) . s\left(v_{i}\right)$ is a strictly decreasing function, that is $s^{\prime}\left(v_{i}\right)<0$, because the attribute is more crucial if it is prior in the sequence; c). $s^{\prime \prime}\left(v_{i}\right)>0$, since the margin sensitivity decreases with the psychological feelings.

Though many kinds of definitions on importance function exist, it seems reasonable as long as it satisfies three conditions above. In this scheme, importance function and weight function are presented in (7), (8).

$$
\begin{gathered}
s\left(v_{i}\right)=a^{v_{i}-1}, a \in(0,1) \\
w\left(v_{i}\right)=\frac{s\left(v_{i}\right)}{s\left(v_{i 1}\right)+s\left(v_{i 2}\right)+s\left(v_{i 3}\right)+s\left(v_{i 4}\right)+s\left(v_{i 5}\right)}
\end{gathered}
$$

Parameter $a$ ranges from 0 to 1 , with $a$ being close to 0 suggests the prior attribute to be more important than the subsequent one and vice versa. In this context, we set $a$ to be 0.8 and show the function in Fig. 1.

So the value of $w(1), w(2), w(3), w(4), w(5)$, can be determined as follows when $a$ is 0.8 .

$$
\begin{aligned}
& s(1)=1.000, w(1)=30 \% \\
& s(2)=0.800, w(2)=24 \% \\
& s(3)=0.640, w(3)=19 \% \\
& s(4)=0.512, w(4)=15 \% \\
& s(5)=0.410, w(5)=12 \% \\
& w\left(v_{i q}\right) \in\{w(1), w(2), w(3), w(4), w(5)\}, \quad q=1,2,3,4,5
\end{aligned}
$$

For example, if the preference order of $F_{i}$ is first location, then rental price, then surroundings, then living space, and the last is floor height, so

$$
\begin{aligned}
& w\left(v_{i 1}\right)=w(1)=30 \% \\
& w\left(v_{i 2}\right)=w(4)=15 \% \\
& w\left(v_{i 3}\right)=w(2)=24 \% \\
& w\left(v_{i 4}\right)=w(5)=12 \% \\
& w\left(v_{i 5}\right)=w(3)=19 \%
\end{aligned}
$$

Given these sub-matching degrees and their weights, the matching degree between applicant family and public housing can be computed according to the definition of $d_{i j}$.

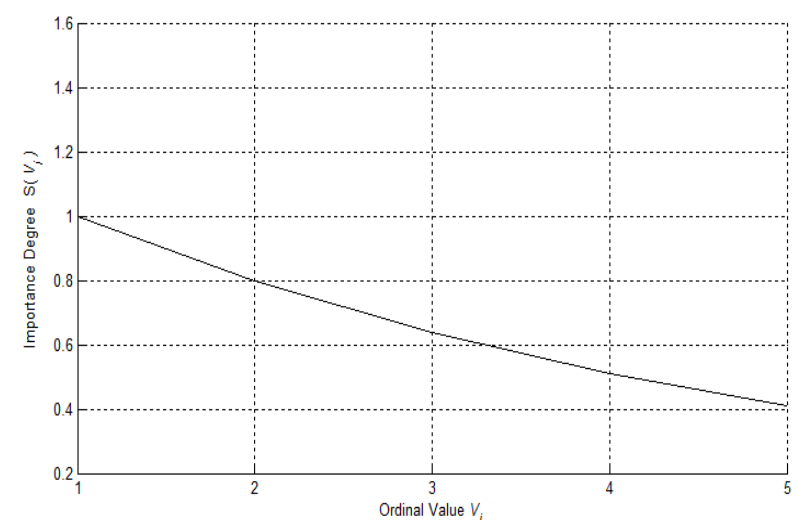

Fig. 1. Graph of importance function.

\section{B. Optimal Objective}

Now there are $m$ applicant families and $n$ suits of public housing. Actually, the amount of applicant family is larger than that of public housing in that the public housing is a kind of scarce and limited resource in China. To conduct an equitable distribution scheme, we should take more other factors into consideration besides matching degree between applicant family and public housing. Applicant family in the group who needs a suit of public housing has different priorities. But which is the first?

There are two different ways to deal with the problem about priority. The first way is to carry out the distribution strictly in accordance with the sequence of applicant family. Among the selectable public housing, a prior applicant family on the family list will get the one with which it has the highest matching degree. Having been assigned to a prior applicant family, this suit of public housing will be deleted from the list of selectable public housing. Then we will have the next applicant family on the list matched with the housing among the new selectable public housing as above.

Assuming that the elements in set of family $F$ have been ordered in sequence, namely, $F_{1}$ has the highest prior, $F_{2}$ has the second highest prior and so on. $M$ is a set of numbers whose elements are infinitely-great positive numbers, and the symbol '>>' means 'far great than' . To seek a total matching degree, labeled as $D$, with the consideration of priority, we construct the model as follows.

$$
\begin{gathered}
\max \quad D=\sum_{i=1}^{m} \sum_{j=1}^{n} M_{i} d_{i j} x_{i j} \\
\text { s.t. } \begin{cases}\sum_{j=1}^{n} x_{i j} \leq 1, \quad i=1,2, \cdots, m \\
\sum_{i=1}^{m} x_{i j} \leq 1, \quad j=1,2, \cdots, n \\
x_{i j}=0 \quad \text { or } \quad 1, \quad i=1,2, \cdots, m ; \quad j=1,2, \cdots, n \\
M=\left\{\begin{array}{lll}
M_{1}, & M_{2}, \quad \cdots \quad, M_{m}
\end{array}\right\}, M_{1}>M_{2}>>>M_{m}\end{cases}
\end{gathered}
$$

If the amount of applicant families is lower than that of public housing, each family can get a housing. Unfortunately this assumption cannot hold in most cases, which means the last $m-n$ applicant families can not obtain a suit of housing 
definitely. The solution in this case seems much easier since we do not have to adopt some special algorithms and only

need one piece of program code about circular sequence.

TABLE II: THE EXPECTATIONS AND PREFERENCE ORDERS OF APPLICANT FAMILIES

\begin{tabular}{llllllll}
\hline \hline $\begin{array}{l}\text { Applicant } \\
\text { family }\end{array}$ & $k_{i}$ & $\alpha_{i}$ & $\beta_{i}$ & $\gamma_{i}$ & $\delta_{i}$ & $\varepsilon_{i}$ & Preference orders \\
\hline$F_{1}$ & 1.45 & $\alpha_{11}=A_{1}, \alpha_{12}=B_{1}, \alpha_{13}=C_{1}$ & $\beta_{11}=45, \beta_{12}=60$ & $\gamma_{1}=300$ & $\delta_{1}=f_{3}$ & $\varepsilon_{1}=\left\{\varepsilon_{11}, \varepsilon_{12}\right\}$ & \\
$F_{2}$ & 1.4 & $\alpha_{21}=A_{2}, \alpha_{22}=B_{3}, \alpha_{23}=C_{3}$ & $\beta_{21}=30, \beta_{22}=45$ & $\gamma_{2}=200$ & $\delta_{2}=f_{4}$ & $\varepsilon_{2}=\left\{\varepsilon_{21}, \varepsilon_{22}, \varepsilon_{23}, \varepsilon_{24}\right\}$ & $\alpha \succ \beta \succ \gamma \succ \delta \succ \varepsilon$ \\
$F_{3}$ & 1.35 & $\alpha_{31}=A_{1}, \alpha_{32}=B_{1}, \alpha_{33}=C_{1}$ & $\beta_{31}=45, \beta_{32}=60$ & $\gamma_{3}=300$ & $\delta_{3}=f_{1}$ & $\varepsilon_{3}=\left\{\varepsilon_{31}, \varepsilon_{32}, \varepsilon_{34}\right\}$ & $\beta \succ \gamma \succ \alpha \succ \delta \succ \varepsilon$ \\
$F_{4}$ & 1.3 & $\alpha_{41}=A_{1}, \alpha_{42}=B_{1}, \alpha_{43}=C_{5}$ & $\beta_{41}=60, \beta_{42}=75$ & $\gamma_{4}=300$ & $\delta_{4}=f_{4}$ & $\varepsilon_{4}=\left\{\varepsilon_{41}, \varepsilon_{42}, \varepsilon_{43}\right\}$ & $\alpha \succ \beta \succ \gamma \succ \varepsilon \succ \delta$ \\
$F_{5}$ & 1.25 & $\alpha_{51}=A_{2}, \alpha_{52}=B_{3}, \alpha_{53}=C_{6}$ & $\beta_{51}=60, \beta_{52}=75$ & $\gamma_{5}=400$ & $\delta_{5}=f_{3}$ & $\varepsilon_{5}=\left\{\varepsilon_{52}, \varepsilon_{53}\right\}$ & $\alpha \succ \gamma \succ \beta \succ \delta \succ \varepsilon$ \\
$F_{6}$ & 1.2 & $\alpha_{61}=A_{3}, \alpha_{62}=B_{4}, \alpha_{63}=C_{4}$ & $\beta_{61}=75, \beta_{62}=+\infty$ & $\gamma_{6}=400$ & $\delta_{6}=f_{2}$ & $\varepsilon_{6}=\left\{\varepsilon_{61}, \varepsilon_{62}, \varepsilon_{63}, \varepsilon_{64}\right\}$ & $\gamma \succ \alpha \succ \beta \succ \varepsilon \succ \delta$ \\
$F_{7}$ & 1.15 & $\alpha_{71}=A_{3}, \alpha_{72}=B_{4}, \alpha_{73}=C_{4}$ & $\beta_{71}=60, \beta_{72}=75$ & $\gamma_{7}=300$ & $\delta_{7}=f_{2}$ & $\varepsilon_{7}=\left\{\varepsilon_{72}, \varepsilon_{73}, \varepsilon_{74}\right\}$ & $\alpha \succ \beta \succ \gamma \succ \delta \succ \varepsilon$ \\
$F_{8}$ & 1.1 & $\alpha_{81}=A_{1}, \alpha_{82}=B_{2}, \alpha_{83}=C_{2}$ & $\beta_{81}=45, \beta_{82}=60$ & $\gamma_{8}=200$ & $\delta_{8}=f_{5}$ & $\varepsilon_{8}=\left\{\varepsilon_{81}, \varepsilon_{82}, \varepsilon_{83}, \varepsilon_{84}\right\}$ & $\gamma \succ \alpha \succ \beta \succ \varepsilon \succ \delta$ \\
$F_{9}$ & 1.05 & $\alpha_{91}=A_{2}, \alpha_{92}=B_{3}, \alpha_{93}=C_{8}$ & $\beta_{91}=60, \beta_{92}=75$ & $\gamma_{9}=300$ & $\delta_{9}=f_{1}$ & $\varepsilon_{9}=\left\{\varepsilon_{91}, \varepsilon_{92}, \varepsilon_{93}, \varepsilon_{94}\right\}$ & $\alpha \succ \beta \succ \gamma \succ \delta \succ \varepsilon$ \\
$F_{10}$ & 1 & $\alpha_{101}=A_{3}, \alpha_{102}=B_{5}, \alpha_{103}=C_{7}$ & $\beta_{101}=15, \beta_{102}=30$ & $\gamma_{10}=100$ & $\delta_{10}=f_{3}$ & $\varepsilon_{10}=\left\{\varepsilon_{103}, \varepsilon_{104}\right\}$ & $\beta \succ \gamma \succ \alpha \succ \delta \succ \varepsilon$ \\
\hline \hline
\end{tabular}

TABLE III: THE REAL VALUE ON CRUCIAL ATtRIBUTES OF PUBLIC HOUSING

\begin{tabular}{llllll}
\hline \hline $\begin{array}{l}\text { Public } \\
\text { housing }\end{array}$ & $a_{j}$ & $b_{j}$ & $c_{j}$ & $d_{j}$ & $e_{j}$ \\
\hline$H_{1}$ & $\alpha_{11}=A_{1}, \alpha_{12}=B_{1}, \alpha_{13}=C_{1}$ & $b_{1}=64$ & $c_{1}=480$ & $d_{1}=f_{3}$ & $e_{11}=90, e_{12}=80, e_{13}=85, e_{14}=90$ \\
$H_{2}$ & $\alpha_{11}=A_{1}, \alpha_{12}=B_{1}, \alpha_{13}=C_{1}$ & $b_{2}=70$ & $c_{2}=530$ & $d_{2}=f_{4}$ & $e_{21}=90, e_{22}=80, e_{23}=85, e_{24}=90$ \\
$H_{3}$ & $\alpha_{11}=A_{1}, \alpha_{12}=B_{2}, \alpha_{13}=C_{2}$ & $b_{3}=52$ & $c_{3}=350$ & $d_{3}=f_{3}$ & $e_{31}=85, e_{32}=70, e_{33}=70, e_{34}=80$ \\
$H_{4}$ & $\alpha_{11}=A_{1}, \alpha_{12}=B_{2}, \alpha_{13}=C_{2}$ & $b_{4}=60$ & $c_{4}=350$ & $d_{4}=f_{2}$ & $e_{41}=85, e_{42}=70, e_{43}=70, e_{44}=80$ \\
$H_{5}$ & $\alpha_{11}=A_{1}, \alpha_{12}=B_{2}, \alpha_{13}=C_{2}$ & $b_{5}=64$ & $c_{5}=420$ & $d_{5}=f_{4}$ & $e_{51}=85, e_{52}=70, e_{53}=70, e_{54}=80$ \\
$H_{6}$ & $\alpha_{11}=A_{2}, \alpha_{12}=B_{3}, \alpha_{13}=C_{3}$ & $b_{6}=52$ & $c_{6}=320$ & $d_{6}=f_{5}$ & $e_{61}=70, e_{62}=70, e_{63}=80, e_{64}=90$ \\
$H_{7}$ & $\alpha_{11}=A_{2}, \alpha_{12}=B_{3}, \alpha_{13}=C_{3}$ & $b_{7}=52$ & $c_{7}=320$ & $d_{7}=f_{4}$ & $e_{71}=70, e_{72}=70, e_{73}=80, e_{74}=90$ \\
$H_{8}$ & $\alpha_{11}=A_{2}, \alpha_{12}=B_{3}, \alpha_{13}=C_{3}$ & $b_{8}=52$ & $c_{8}=300$ & $d_{8}=f_{5}$ & $e_{81}=70, e_{82}=70, e_{83}=80, e_{84}=90$ \\
$H_{9}$ & $\alpha_{11}=A_{3}, \alpha_{12}=B_{4}, \alpha_{13}=C_{4}$ & $b_{9}=42$ & $c_{9}=280$ & $d_{9}=f_{1}$ & $e_{91}=80, e_{92}=80, e_{93}=85, e_{94}=80$ \\
$H_{10}$ & $\alpha_{11}=A_{3}, \alpha_{12}=B_{4}, \alpha_{13}=C_{4}$ & $b_{10}=30$ & $c_{10}=245$ & $d_{10}=f_{5}$ & $e_{101}=80, e_{102}=80, e_{103}=85, e_{104}=80$ \\
\hline \hline
\end{tabular}

The other way is to assign a priority index to each member in the group. The priority index just likes weight, with the applicant family on the top of the list having a higher priority index. We label the priority index of family $F_{i}$ as $k_{i}$, the maximum of priority index as $k_{\max }$, and the minimum value as $k_{\min } . u_{i}$ is the ordinal value of family $F_{i}$ on the families list. Ordinal value of the first one on the list is 1 , and ordinal value of the last one on the list is $m$. And $k_{i}$ is presented in (9).

$$
k_{i}=k_{\max }-\frac{k_{\max }-k_{\min }}{m-1} \times\left(u_{i}-1\right), \quad u_{i}=1,2,3, \cdots, m
$$

Given the priority index of applicant family, we can model the distribution of public housing as an assignment problem, which is one of the fundamental combinatorial optimization problems in the branch of optimization or operations research. The model is shown as follows.

$$
\max \quad D=\sum_{i=1}^{m} \sum_{j=1}^{n} k_{i} d_{i j} x_{i j}
$$

In this assignment model, there are $n$ suits of public housing and $m$ applicant families in the public housing distribution process. Unlike the matching mode in the
E-commerce or stock trading, each applicant family can only obtain one suit of public housing, and each public housing is only given to a certain family. The optimal objective of the assignment model is to maximum the total matching degree. Considering that members on the family list are heterogeneous in priority indexes, the total matching degree of applicant families is the sum of an applicant family's matching degree to the public housing multiples its priority index.

$$
\text { s.t. }\left\{\begin{array}{l}
\sum_{j=1}^{n} x_{i j} \leq 1, \quad i=1,2, \cdots, m \\
\sum_{i=1}^{m} x_{i j} \leq 1, \quad j=1,2, \cdots, n \\
x_{i j}=0 \quad \text { or } \quad 1, \quad i=1,2, \cdots, m ; \quad j=1,2, \cdots, n
\end{array}\right.
$$

As one of many algorithms for solving the linear assignment problem within time bounded by a polynomial expression of the number of agents, Hungarian algorithm has been extensively discussed in [4]. We can also transform this model into a maximum weight matching problem in the graphic theory and solve it by using Kuhn-Munkres algorithm, which is discussed in [5], [6].

\section{Algorithm Instance}


TABLE IV: MATCHING DEGREE AND WEIGHTED MATCHING DEGREE

\begin{tabular}{|c|c|c|c|c|c|c|c|c|c|c|}
\hline $\begin{array}{l}d_{i j} \\
k_{i} d_{i j}\end{array}$ & 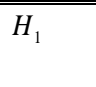 & $\overline{\mathrm{CH}}$ & $\overline{\mathrm{CH}}$ & $\mathrm{H}_{4}$ & 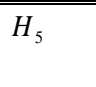 & $\overline{H_{6}}$ & 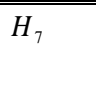 & 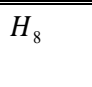 & 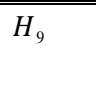 & $\overline{H_{10}}$ \\
\hline \multirow[t]{2}{*}{$F_{1}$} & 90.10 & 82.20 & 86.40 & 80.40 & 76.10 & 68.45 & 71.45 & 69.40 & 67.00 & 63.40 \\
\hline & 130.65 & 119.19 & 125.28 & 116.58 & 110.35 & 99.25 & 103.60 & 100.63 & 97.15 & 91.53 \\
\hline \multirow[t]{2}{*}{$F_{2}$} & 58.05 & 64.05 & 64.05 & 61.05 & 67.65 & 87.60 & 90.60 & 87.60 & 68.35 & 75.55 \\
\hline & 81.27 & 89.67 & 89.67 & 85.47 & 94.71 & 122.64 & 126.84 & 122.64 & 95.69 & 105.77 \\
\hline \multirow[t]{2}{*}{$F_{3}$} & 79.20 & 76.80 & 80.30 & 86.30 & 74.90 & 75.60 & 75.60 & 76.80 & 81.70 & 68.20 \\
\hline & 106.92 & 103.68 & 108.41 & 116.51 & 101.12 & 102.06 & 102.06 & 103.68 & 110.30 & 92.07 \\
\hline \multirow[t]{2}{*}{$F_{4}$} & 84.25 & 87.15 & 75.75 & 78.15 & 83.45 & 69.85 & 72.25 & 70.80 & 64.85 & 62.45 \\
\hline & 109.53 & 113.30 & 98.48 & 101.60 & 108.49 & 90.81 & 93.93 & 92.04 & 84.31 & 81.19 \\
\hline \multirow[t]{2}{*}{$F_{5}$} & 77.50 & 69.10 & 74.60 & 72.40 & 71.20 & 81.20 & 84.20 & 81.20 & 65.20 & 59.50 \\
\hline & 96.88 & 86.38 & 93.25 & 90.50 & 89.00 & 101.50 & 105.25 & 101.50 & 81.5 & 74.38 \\
\hline \multirow[t]{2}{*}{$F_{6}$} & 70.99 & 66.54 & 69.64 & 76.34 & 68.59 & 67.43 & 67.43 & 67.43 & 83.39 & 78.59 \\
\hline & 85.19 & 79.85 & 83.57 & 91.61 & 82.31 & 80.92 & 80.92 & 80.92 & 100.07 & 94.31 \\
\hline \multirow[t]{2}{*}{$F_{7}$} & 68.50 & 63.60 & 66.10 & 76.90 & 66.00 & 64.85 & 64.85 & 65.80 & 87.60 & 74.40 \\
\hline & 78.78 & 73.14 & 76.02 & 88.44 & 75.90 & 74.58 & 74.58 & 75.67 & 100.74 & 85.56 \\
\hline \multirow[t]{2}{*}{$F_{8}$} & 63.64 & 68.44 & 80.24 & 80.24 & 80.14 & 76.23 & 73.83 & 76.23 & 69.74 & 75.59 \\
\hline & 70.00 & 75.28 & 88.26 & 88.26 & 88.15 & 83.85 & 81.21 & 83.85 & 76.71 & 83.15 \\
\hline \multirow[t]{2}{*}{$F_{9}$} & 65.65 & 63.75 & 63.45 & 74.25 & 66.35 & 79.55 & 79.55 & 80.50 & 72.55 & 56.35 \\
\hline & 68.93 & 66.94 & 66.62 & 77.96 & 69.67 & 83.53 & 83.53 & 84.53 & 76.18 & 59.17 \\
\hline \multirow[t]{2}{*}{$F_{10}$} & 69.70 & 63.70 & 68.20 & 62.20 & 62.20 & 65.20 & 68.20 & 65.20 & 73.00 & 78.40 \\
\hline & 69.70 & 63.70 & 68.20 & 62.20 & 62.20 & 65.20 & 68.20 & 65.20 & 73.00 & 78.40 \\
\hline
\end{tabular}

In this section, we collect some actual data of applicant family and public housing in Huangshi and use two different ways to conduct the distribution. Specifically, we list ten suit of public housing and ten applicant families as well as their expectable values of five crucial attributes and preference orders. Let $k_{\max }=1.45$ and $k_{\min }=1$, and then order the set of family $F$ based on their priority in the sequence so that the values of $k_{1} \sim k_{10}$ range from 1.45 to 1 . Simply, $\alpha, \beta, \gamma, \delta, \varepsilon$ represent the attributes of location, living space, rental price, floor height and surroundings respectively, and ' $\succ$ ' means 'more preferential'. For example, if one applicant family's preference order is that the first preference is location, the next living space, then rental price, then floor height and then surroundings, we represent it as $\alpha \succ \beta \succ \gamma \succ \delta \succ \varepsilon$. Due to the difficulty in recording address names, we use $A_{1}$ to $A_{3}, B_{1}$ to $B_{5}$ and $C_{1}$ to $C_{8}$ to present the names of districts, sub-districts and communities. We show the information of ten applicant families in Table II, and the real values of five crucial attributes of public housing in Table III.

With reference to its definition, the matching degree between the family and the housing can be obtained by weighted sum of each sub-matching degree. Take $d_{11}$ for example: as the expectations of district, sub-district and community of $F_{1}$ are the same with the real values of them of $H_{1}$ exactly, the sub-matching degree of location is 100; the real value of living space is larger than the right boundary value of its expectation, so the sub-matching degree of living space equals to 90 ; the expectation of rental price is 300 , whereas the real rental price is 480 , resulting in the sub-matching degree of rental price being 70; both of the expectation and real value of floor height is medium floor, which suggests the sub-matching degree equal to 100; and this family choose educational and medical facilities as their preference on surrounding, and the real values are 90 and 80 , so the sub-matching degree of its surroundings is 85 . According to its preference order, the weights of location, living space, rental price, floor height and surroundings are $30 \%, 24 \%, 19 \%, 15 \%$ and $12 \%$ respectively. Then $d_{11}$ is 90.1 , computed by weighted sum of sub-matching degree. The matching degree and weighted matching degree are shown in Table IV, and the matching outcomes through two different ways are given in Table $\mathrm{V}$.

TABLE V: MATCHING OUTCOMES THROUGH Two WAYS

\begin{tabular}{cll}
\hline \hline $\max D=\sum_{i=1}^{m} \sum_{j=1}^{n} M_{i} d_{i j} x_{i j}$ & $\max$ & $D=\sum_{i=1}^{m} \sum_{j=1}^{n} k_{i} d_{i j} x_{i j}$ \\
\hline$F_{1} \sim H_{1}$ & $F_{1} \sim H_{1}$ \\
$F_{2} \sim H_{7}$ & $F_{2} \sim H_{8}$ \\
$F_{3} \sim H_{4}$ & $F_{3} \sim H_{3}$ \\
$F_{4} \sim H_{2}$ & \\
$F_{5} \sim H_{6}$ & $F_{4} \sim H_{2}$ \\
$F_{6} \sim H_{9}$ & $F_{5} \sim H_{7}$ \\
$F_{7} \sim H_{10}$ & $F_{6} \sim H_{5}$ \\
$F_{8} \sim H_{3}$ & $F_{7} \sim H_{9}$ \\
$F_{9} \sim H_{8}$ & $F_{8} \sim H_{6}$ \\
$F_{10} \sim H_{5}$ & $F_{9} \sim H_{4}$ \\
\hline \hline
\end{tabular}




\section{CONCLUSION}

This article designs a scheme on the distribution of public housing in Huangshi, China. We identify five crucial attributes of public housing: location, living space, rental price, floor height and surroundings. The expectation and preference order of these attributes are believed to represent the housing preference of applicant family. We compute the matching degree by comparing expectations of applicant family and real values of public housing. Finally, this paper proposes two ways of distribution. The first one comes strictly from ordinal position of applicant family, whereas the second one maximizes a total matching degree by taking each applicant family's priority index as weight factor. The scheme has been carry out in Huangshi, and the following research attempts to complement the computational formula of sub-matching degree and adjust the weights according to the local real situation.

\section{ACKNOWLEDGMENT}

The authors wish to thank the support from the Real Estate Board of Huangshi.

\section{REFERENCES}

[1] Annual Statistical Bulletin of the National Bureau of Statistics. [Online]. Available: http://www.stats.gov.cn/tjgb/.

[2] The Twelfth Five-year Plan for National Economic and Social Development of the People's Republic of China. [Online]. Available: http://news.xinhuanet.com/politics/2011-03/16/c_121193916.htm

[3] D. Gale and L. S. Shapley, "College admissions and the stability of marriage," American Mathematical Monthly, vol. 69, no. 1, pp. 9-15, 1962.
[4] H. W. Kuhn, "The Hungarian method for the assignment problem," Naval Research Logistics Quarterly, vol. 2, pp. 83-97. 1955.

[5] J. Edmonds, "Maximum matching and a polyhedron With 0,1-Vertices," Journal of Research of the National Bureau of Standards, vol. 69, pp. 125-130, 1965.

[6] J. A. Bondy and U. S. R. Murty, Graph Theory with Applications: London: The Macmillan Press Ltd, 1976, ch. 5.

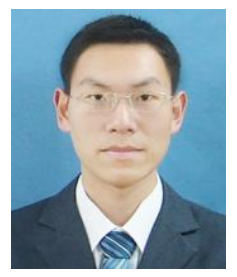

Xiao Liu was born in Hubei, China. He received his bachelor's degree in information management and information system from Huazhong University of Science and Technology. He is currently a PhD student in management science and engineering at Huazhong University of Science and Technology. His major areas of research are intelligent business, information planning and IT project management.

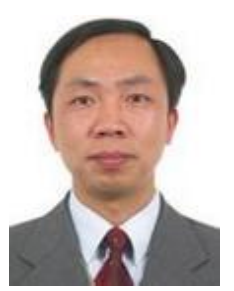

Huimin Ma was born in Hubei, China. He received his $\mathrm{PhD}$ degree from Huazhong University of Science and Technology. He is currently an associate professor at department of management science and information system, Huazhong University of Science and Technology. His major areas of research are housing management, information planning, E-commerce and data mining.

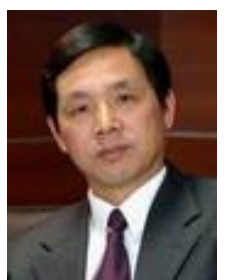

Jinlong Zhang was born in Jiangxi, China. He received his $\mathrm{PhD}$ degree from Huazhong University of Science and Technology. He is currently a professor at department of management science and information system, Huazhong University of Science and Technology. His major areas of research are information management, E-commerce, logistic management and management innovation. 